\title{
APPLICATIONS OF MAX ALGEBRA TO DIAGONAL SCALING OF MATRICES*
}

\author{
PETER BUTKOVIC ${ }^{\dagger}$ AND HANS SCHNEIDER ${ }^{\ddagger}$
}

Dedicated to Pauline van den Driessche on the occasion of her 65 th birthday in appreciation of her contributions to the fields of linear algebra and mathematical biology.

\begin{abstract}
Results are proven on an inequality in max algebra and applied to theorems on the diagonal similarity scaling of matrices. Thus the set of all solutions to several scaling problems is obtained. Also introduced is the "full term rank" scaling of a matrix to a matrix with prescribed row and column maxima with the additional requirement that all the maxima are attained at entries each from a different row and column. An algorithm which finds such a scaling when it exists is given.
\end{abstract}

Key words. Max algebra inequality, Diagonal scaling, Term rank scaling, Algorithm.

AMS subject classifications. 15A24, 15A15, 15A48, 90C27.

1. Introduction. The aim of this paper is to prove results on an inequality in max algebra and to apply these to diagonal similarity scaling problems for matrices. Thus we trace a connection between these fields.

Let $a \oplus b=\max (a, b)$ and $a \otimes b=a . b$ for non-negative real numbers $a$ and $b$. By max algebra we understand the analogue of linear algebra developed for the pair of operations $(\oplus, \otimes)$ extended to matrices and vectors formally in the same way as in linear algebra.

Motivated by a range of applications, many authors have considered max algebra in the past. Although the first papers related to max algebra appeared as early as in the 1950's or perhaps even before, the first comprehensive monograph seems to be [7] which was later extended and updated [8]. Many algebraic and algorithmic aspects were studied [24]. Other relevant sources are: [1] with many applications and various different approaches, papers [17], [18], [4], [9], [10], and the thesis [15]. Combinatorial aspects are studied in [6]. A summer school's proceedings [16] is a collection of a number of the state-of-the-art papers including a thorough historical overview of the field. A chapter on max algebra can be found in [2].

Quite independently, matrix diagonal scaling has been intensively studied by a number of authors [13], [14], [12], [11], [23], [19] and others. A diagonal similarity scaling of a square real matrix $A$ is a matrix of the form $X^{-1} A X$ where $X$ is a diagonal matrix with positive diagonal. Among other problems, the papers mentioned above considered and solved the following similarity diagonal scaling problem and some

*Received by the editors 3 August 2005. Accepted for publication on 30 October 2005. Handling Editor: Ravindra B. Bapat. Supported by the EPSRC grant T06414.

$\dagger$ School of Mathematics, University of Birmingham, Edgbaston, Birmingham B15 2TT, UK (p.butkovic@bham.ac.uk).

$\ddagger$ Department of Mathematics, University of Wisconsin-Madison, Madison, WI 53706, USA (hans@math.wisc.edu). 
generalizations.

Problem 1.1. Given a nonnegative square matrix $A$ and a positive constant $\mu$ find a diagonal matrix $X$ with positive diagonal such that $X^{-1} A X \leq \mu E$, where $E$ is the matrix whose every entry is 1.

We shall provide a simple proof of a solution using max algebra in which we add the description of all matrices $X$ that solve the above problem. The importance of this problem lies in the fact that many other scaling problems can be reduced to it, see, e.g. [19]. Some examples are given in the current paper.

In Section 2 we first recall basic concepts of graph theory and max algebra and then we determine the solution set of the inequality $A \otimes x \leq \mu \otimes x$ for $\mu>0$ and $x>0$ in max algebra. Our main emphasis is the use of a matrix $\Delta(A)$ which is the cumulative max algebra sum of powers of $A$. In Section 3 we then apply our results to several scaling problems which have been previously considered. Our method yields a complete description of the set of $X$ which solve the desired scaling. In Section 4 we apply our results to a newly defined type of scaling ("full term rank scaling"). We produce a theoretical solution and show how it may be obtained by an algorithm.

We note that, for the sake of simplicity, in Problem 1.1 we speak of finding a solution when in fact we intend to determine necessary and sufficient conditions for a solution of the problem to exist and, if these are satisfied, then to find a solution or even all solutions, either in the form of a theorem or by an algorithm. We follow this style in the statement of subsequent problems.

2. Max algebra basics and results. We summarize basic concepts and results in max algebra [7], [8], [1] which will be useful later on in the paper. The symbol $\mathbb{R}_{+}$will denote the set of non-negative real numbers. In the whole paper $n \geq 1$ is an integer and $N=\{1, \ldots, n\}$. If $x=\left(x_{1}, \ldots, x_{n}\right)^{T} \in \mathbb{R}_{+}^{n}$ then $x>0$ means that $x_{j}>0$ for all $j \in N$.

For non-negative real numbers $a, b$ we define $a \oplus b:=\max (a, b), a \otimes b:=a . b$.

If $A=\left(a_{i j}\right), B=\left(b_{i j}\right)$ and $C=\left(c_{i j}\right)$ are non-negative matrices of compatible sizes and $\alpha$ is a non-negative real number, we write $C=A \oplus B$ if $c_{i j}=a_{i} \oplus b_{i j}$ for all $i, j, C=A \otimes B$ if $c_{i j}=\sum_{k}^{\oplus} a_{i k} \otimes b_{k j}=\max _{k}\left(a_{i k} b_{k j}\right)$ for all $i, j$ and $C=\alpha \otimes A$ if $c_{i j}=\alpha \otimes \alpha_{i j}$ for all $i, j$. It is easily proved that $A \leq B$ implies that $A \otimes C \leq B \otimes C$ for any matrices $A, B, C$ of compatible sizes.

So, for an $n \times n$ matrix $A$

$$
A \otimes x \leq \mu \otimes x
$$

means

$$
\max _{j=1, \ldots, n} a_{i j} x_{j} \leq \mu x_{i} \quad(i=1, \ldots, n) .
$$

We may now translate Problem 1.1 into the language of max algebra:

PROBlem 2.1. Given a nonnegative square matrix $A$ and a positive constant $\mu$, find a positive vector $x$ such that $A \otimes x \leq \mu \otimes x$.

If $A=\left(a_{i j}\right) \in \mathbb{R}_{+}^{n \times n}$, then $\mathbf{D}(A)$ will denote the arc-weighted digraph with the node set $N$, arc set $\left\{(i, j) ; a_{i j}>0\right\}$ and arc weights $a_{i j}$. A path in a digraph is 
any finite sequence of nodes $\left(i_{1}, i_{2}, \ldots\right)$ such that $\left(i_{r}, i_{r+1}\right)$ is an $\operatorname{arc}(r=1,2, \ldots)$. If $\sigma=\left(i_{1}, \ldots, i_{k}, i_{k+1}\right)$ is a path in $\mathbf{D}(A)$, then the weight of $\sigma$ is

$$
w(\sigma, A)=a_{i_{1} i_{2}} a_{i_{2} i_{3}} \ldots a_{i_{k} i_{k+1}}
$$

and the length of $\sigma$ is $l(\sigma)=k$. If $i_{k+1}$ and $i_{1}$ coincide then $\sigma$ is called a cycle. If $\sigma=\left(i_{1}, \ldots, i_{k}, i_{1}\right)$ is a cycle in $\mathbf{D}(A)$ then the cycle mean of $\sigma$ is

$$
\mu(\sigma, A)=\sqrt[l(\sigma)]{w(\sigma, A)} .
$$

The maximum cycle mean value is a very important characteristic of matrices in both diagonal scaling and max algebra and is denoted by $\lambda(A)$. It is easily proved that $\lambda(\alpha \otimes A)=\alpha \otimes \lambda(A)$ for every $\alpha>0$.

Note that there are many efficient methods for finding the maximum cycle mean of a matrix, one of them is Karp's algorithm [20] of computational complexity $O\left(n^{3}\right)$.

The iterated product $A \otimes A \otimes \ldots \otimes A$ in which the symbol $A$ appears $k$-times will be denoted by $A^{(k)}$ and we define

$$
\begin{aligned}
\Gamma(A) & =A \oplus A^{(2)} \oplus \cdots \oplus A^{(n)}, \\
\Delta(A) & =I \oplus \Gamma(A),
\end{aligned}
$$

where $I$ is the usual $n \times n$ identity matrix. It is easily seen that $\Delta(A)=\Gamma(I \oplus A)$. Since the $(i, j)$ element of $A^{(k)}$ is the maximum weight of a path in $\mathbf{D}(A)$ from $i$ to $j$ of length $k$ we immediately have the following observations which we collect in a lemma. For $x \in \mathbb{R}_{+}^{n}$, $\operatorname{diag}(x)$ denotes the diagonal matrix in $\mathbb{R}_{+}^{n \times n}$ whose diagonal is $x$, while for $A \in \mathbb{R}_{+}^{n \times n} \operatorname{diag}(A)$ denotes the diagonal matrix $\operatorname{diag}\left(\left[a_{11}, \ldots, a_{n n}\right]\right)$.

Lemma 2.2. Let $A \in \mathbb{R}_{+}^{n \times n}$.

1. If $\lambda(A) \leq 1$ then

$$
\Gamma(A)=A \oplus A^{(2)} \oplus A^{(3)} \oplus \cdots \oplus A^{(k)} \quad \text { for all } \quad k \geq n
$$

and $\Delta(A)$ is idempotent.

2. If $\lambda(A)>1$ then the sequence $A, A^{(2)}, A^{(3)}, \ldots$ is unbounded.

3. $\operatorname{diag}(\Delta(A)) \leq I \Longleftrightarrow \operatorname{diag}(\Delta(A))=I \Longleftrightarrow \lambda(A) \leq 1$.

Here boundedness is taken in any (and therefore every) norm on $\mathbb{R}^{n \times n}$.

LEMMA 2.3. Let $A$ be a nonnegative square matrix. Then $x$ satisfies $A \otimes x \leq x$ if and only if $\Delta(A) \otimes x=x$.

Proof. Suppose that $A \otimes x \leq x$. Then $(I \oplus A) \otimes x=x$ and it follows that $(I \oplus A)^{(k)} \otimes x=x$ for $k=1, \ldots$ Hence also $\Delta(A) \otimes x=\Gamma(I \oplus A) x=x$.

Conversely, if $\Delta(A) \otimes x=x$ then $A \otimes x \leq \Delta(A) \otimes x=x$. $\mathrm{Q}$

We now examine the impact of the condition $\lambda(A) \leq 1$.

Lemma 2.4. Let $A$ be a nonnegative square matrix and let $\lambda(A) \leq 1$. If $u \in \mathbb{R}_{+}^{n}$ then $x=\Delta(A) \otimes u$ satisfies $A \otimes x \leq x$.

Proof. Since $\lambda(A) \leq 1$, we have by Lemma 2.2 that $A \otimes \Delta(A)=\Gamma(A) \leq \Delta(A)$ and the result follows.

We next prove a Proposition of some independent interest which will be referred to 
in a subsequent remark. We put $e^{(i)}$ to be the $i$-th unit vector with entry 1 in the $i$-th position and 0 elsewhere.

Proposition 2.5. Let $A \in \mathbb{R}_{+}^{n \times n}$ and let $\lambda(A) \leq 1$. Then the following are equivalent.

1. For all $x \in \mathbb{R}_{+}^{n}, \quad A \otimes x \leq x \Longrightarrow A \otimes x=x$.

2. $\Gamma(A)=\Delta(A)$.

Proof. 1. $\Longrightarrow 2$. If (1) holds, then by Lemma 2.4 with $x=\Delta(A) \otimes e^{(i)}$ we have

$$
\Delta(A) \otimes e^{(i)}=A \otimes \Delta(A) \otimes e^{(i)}=\Gamma(A) \otimes e^{(i)} .
$$

Since this holds for $i=1, \ldots, n$, we obtain $\Gamma(A)=\Delta(A)$.

2. $\Longrightarrow 1$. Let $\Gamma(A)=\Delta(A)$ and let $A \otimes x \leq x$. Then, since $\lambda(A) \leq 1$ it follows from Lemma 2.2 that

$$
A \otimes x=A \otimes \Delta(A) \otimes x=\Gamma(A) \otimes x=\Delta(A) \otimes x=x,
$$

where the first and last equality hold by Lemma 2.3 .

We now consider $x>0$ and we put $e=[1,1, \ldots, 1]^{t} \in \mathbb{R}_{+}^{n}$.

THEOREM 2.6. Let $A$ be a nonnegative square matrix and let $\mu$ be a positive constant. The following conditions are equivalent.

1. $\lambda(A) \leq \mu$.

2. If $u \geq 0$ and $x:=\Delta(A / \mu) \otimes u$ then

$$
\Delta(A / \mu) \otimes x=x .
$$

3. If $u>0$ then $x:=\Delta(A / \mu) \otimes u$ is positive and satisfies (2.3).

4. There exists a positive vector $x$ that satisfies (2.3).

5. There exists a positive vector $x$ such that

$$
A \otimes x \leq \mu \otimes x
$$

Proof. Replacing $A$ by $A / \mu$ we may assume that $\mu=1$ in the proof below.

$(1) \Longrightarrow(2)$. By Lemmas 2.4 and 2.3 .

$(2) \Longrightarrow(3)$. We note that $x=\Delta(A) \otimes u \geq u>0$ and the result follows.

$(3) \Longrightarrow(4)$. Obvious.

$(4) \Longrightarrow(5)$. By Lemma 2.3.

$(5) \Longrightarrow(1)$. Suppose that $x$ satisfies (5) and note that $e^{(i)} \leq x / c$ where $0<c:=$ $\min _{j} x_{j}$. Thus it follows from (5), that

$$
A^{(k)} \otimes e^{(i)} \leq A^{(k)} \otimes(x / c) \leq x / c, \quad k=1,2 \ldots
$$

Since this holds for $i=1, \ldots, n$, we obtain 1 . from Lemma $2.2 \mathrm{\square}$

REMARK 2.7. Thus in order to check the existence of a positive $x \in \mathbb{R}_{+}^{n}$ that satisfies (2.4) and to find one such vector we need only check that $x:=\Delta(A / \mu) \otimes u$ with $u>0$ satisfies this inequality for an arbitrarily chosen $u>0$, see Theorem 2.6(3). It may be computationally better not to compute $\Delta(A / \mu)$ for this purpose, instead put $x^{(0)}=u$ and then iteratively $x^{(k)}=(A / \mu) \otimes x^{(k-1)}, k=1, \ldots, n$. Then $x^{(n)}=\Delta(A / \mu) \otimes u$. Note that it is also unnecessary to find $\lambda(A)$ explicitly. 
Remark 2.8. Since Problem 2.1 is equivalent to Problem 1.1, we note that the equivalence of Conditions 1. and 5. of Theorem 2.6 is the content of Theorem 7.2 and Remark 7.3 in [12]. This result may also be derived from an extensive theory of max eigenvalues and eigenvectors found in several places, e.g. [7], [8], [15, Chapter 4] and [2].

We now sum up our results in the following Corollary. In order to state it, we define two sets of positive vectors associated with $A \in \mathbb{R}_{+}^{n \times n}$,

$$
\begin{gathered}
\operatorname{Sol}^{+}(A)=\{x>0: A \otimes x \leq x\}, \\
\operatorname{Comb}^{+}(\Delta(A))=\left\{x>0: x=\Delta(A) \otimes u \text { for some } u \in \mathbb{R}_{+}^{n}\right\},
\end{gathered}
$$

viz. $\operatorname{Comb}^{+}(\Delta(A))$ is the set of all max combinations of the columns of $\Delta(A)$ that are positive. Our corollary corresponds to the case $\mu=1$ in Theorem 2.6. To obtain the general case of $\mu>0$ replace $A$ by $A / \mu$. We here emphasize that all needed information is encoded in $\Delta(A)$.

Corollary 2.9. Let $A \in \mathbb{R}_{+}^{n \times n}$. The following are equivalent:

1. $\lambda(A) \leq 1$,

2. $\operatorname{diag}(\Delta(A))=I$,

3. $\operatorname{Sol}^{+}(A) \neq \emptyset$,

4. $\mathrm{Sol}^{+}(A)=\mathrm{Comb}^{+}(\Delta(A))$.

Proof. (1) $\Longleftrightarrow$ (2). By Lemma 2.2 (3).

$(1) \Longleftrightarrow(3)$. By the equivalence of (1) and (5) in Theorem 2.6.

$(4) \Longrightarrow(3)$. Clear, since $\mathrm{Comb}^{+}(\Delta(A)) \neq \emptyset$.

$(3) \Longrightarrow(4)$. Suppose $\operatorname{Sol}^{+}(A) \neq \emptyset$. If $x \in \operatorname{Sol}^{+}(A)$ (viz. $A \otimes x \leq x$ ) then it follows from Lemma 2.3 that $x=\Delta(A) \otimes x$ and so $x \in \operatorname{Comb}^{+}(\Delta(A))$. To show the reverse inclusion, note that Theorem 2.6 (5) holds, since $\operatorname{Sol}^{+}(A) \neq \emptyset$. Hence, if $x \in$ $\mathrm{Comb}^{+}(\Delta(A))$, we apply Theorem $2.6(2)$ and Lemma 2.3 to obtain $x \in \mathrm{Sol}^{+}(A)$.

3. Applications to diagonal similarity scaling. The equivalence of (1) and (5) of Theorem 2.6 is the content of Theorem 7.2 and Remark 7.3 of [12]. Thus we have given a simple max algebra proof of that scaling result. In fact, our proof yields more for we may apply Corollary 3.5 to obtain all solutions of Problem 1.1. We can apply our result to the problems stated below. The fourth of these was considered in [19] who proved a necessary and sufficient condition for the existence of a solution and a method to compute it. The first three problems are special cases of the last one and were solved in some of the references given in the introduction, see [19] for more information. Our techniques allow us to describe efficiently all solutions of these problems in a concise form. For $A, B \in \mathbb{R}_{+}^{n \times n}$ the inclusion $\mathbf{D}(A) \subseteq \mathbf{D}(B)$ is defined to mean the inclusion of the corresponding arcsets: $\left\{(i, j): a_{i j}>0\right\} \subseteq\left\{(i, j): b_{i j}>0\right\}$.

Problem 3.1. Given $A, B \in \mathbb{R}_{+}^{n \times n}$ with $\mathbf{D}(A) \subseteq \mathbf{D}(B)$, find a diagonal matrix $X$ with positive diagonal so that

$$
X^{-1} A X \leq B
$$

where $X^{-1} A X$ is the ordinary matrix product. 
Problem 3.2. Given $A, B \in \mathbb{R}_{+}^{n \times n}$ with $\mathbf{D}(A)=\mathbf{D}(B)$, find a diagonal matrix $X$ with positive diagonal so that

$$
X^{-1} A X=B
$$

Problem 3.3. Given $A, B, C \in \mathbb{R}_{+}^{n \times n}$ with $\mathbf{D}(C) \subseteq \mathbf{D}(A) \subseteq \mathbf{D}(B)$, find a diagonal matrix $X$ with positive diagonal so that

$$
C \leq X^{-1} A X \leq B
$$

Problem 3.4. Given $A^{(k)}, B^{(k)}, C^{(k)} \in \mathbb{R}_{+}^{n \times n}$ with $\mathbf{D}\left(C^{(k)}\right) \subseteq \mathbf{D}\left(A^{(k)}\right) \subseteq$ $\mathbf{D}\left(B^{(k)}\right), k=1, \ldots, s$, find a diagonal matrix $X$ with positive diagonal so that $C^{(k)} \leq$ $X^{-1} A^{(k)} X \leq B^{(k)}$ for $k=1, \ldots, s$.

We note that Problem 3.3 is obtained from Problem 3.4 by putting $k=1$ and then Problems 3.2 and 3.1 are obtained from Problem 3.3 by putting $C=B$ and $C=0$ respectively.

Suppose that $\mathbf{D}(C) \subseteq \mathbf{D}(A) \subseteq \mathbf{D}(B)$. First, it is easily seen that $X^{-1} A X \leq B$ is equivalent to $(A / B) \otimes x \leq x$, where $A / B$ is defined by

$$
\begin{aligned}
(A / B)_{i j} & =a_{i j} \otimes b_{i j}^{-1} \quad \text { if } \quad b_{i j} \neq 0 \\
& =0 \quad \text { if } \quad b_{i j}=0 .
\end{aligned}
$$

Second, we have

$$
\begin{aligned}
X^{-1} A X & \geq C \Longleftrightarrow X C X^{-1} \leq A \\
& \Longleftrightarrow X^{-1} C^{T} X \leq A^{T} \\
& \Longleftrightarrow X^{-1}\left(C^{T} / A^{T}\right) X \leq E \\
& \Longleftrightarrow\left(C^{T} / A^{T}\right) \otimes x \leq x .
\end{aligned}
$$

Third, note that $x$ is a solution to $A \otimes x \leq x$ and $B \otimes x \leq x$ if and only if it is a solution to $(A \oplus B) \otimes x \leq x$. Of course, this remark extends to a sequence of matrices.

We can now apply these observations to Corollary 2.9 to add information to known results. We also replace Condition (1) of Theorem 2.6 by the equivalent form given in Lemma $2.2(3)$ in order to stress that all the information we need is encoded in $\Delta(Q)$ for a well-chosen matrix $Q$.

TheOREM 3.5. Suppose that $A^{(k)}, B^{(k)}, C^{(k)} \in \mathbb{R}_{+}^{n \times n}$ with $\mathbf{D}\left(C^{(k)}\right) \subseteq \mathbf{D}\left(A^{(k)}\right) \subseteq$ $\mathbf{D}\left(B^{(k)}\right), k=1, \ldots, s$. Let

$$
Q=\Sigma_{k}^{\oplus} A^{(k)} / B^{(k)} \oplus \Sigma_{k}^{\oplus} C^{(k) T} / A^{(k) T} .
$$

Then, for Problem 3.4, the following are equivalent:

1.

$$
\operatorname{diag}(\Delta(Q))=I
$$


2. There exists a solution to the problem.

3. The matrix $X=\operatorname{diag}(x)$ with $x>0$ solves the problem if and only if $x \in$ $\mathrm{Comb}^{+}(\Delta(Q))$.

We note that the equivalence of (1) and (2) in Theorem 3.5 can be found in [19]. Putting $k=1$ in Theorem 3.5 and then $C=B$ or $C=0$ we obtain the following Corollary.

Corollary 3.6. Suppose that $A, B, C \in \mathbb{R}_{+}^{n \times n}$ with $\mathbf{D}(C) \subseteq \mathbf{D}(A) \subseteq \mathbf{D}(B)$. Then, for Problems 3.1, 3.2 and 3.3, conditions (1), (2) and (3) of Theorem 3.5 are equivalent, where

- for Problem 3.1,

$$
Q=A / B
$$

- for Problem 3.2,

$$
Q=B^{T} / A^{T} \oplus A / B
$$

- and for Problem 3.3

$$
Q=C^{T} / A^{T} \oplus A / B
$$

EXAMPLE 3.7. Let

$$
A=\left[\begin{array}{lll}
2 & 0 & 0 \\
9 & 1 & 2 \\
3 & \frac{1}{2} & 0
\end{array}\right], \quad B=\left[\begin{array}{lll}
3 & 0 & 1 \\
3 & 1 & 3 \\
3 & 1 & 0
\end{array}\right], \quad C=\left[\begin{array}{lll}
1 & 0 & 0 \\
1 & 1 & 0 \\
1 & \frac{2}{3} & 0
\end{array}\right]
$$

and consider Problem 3.3. We have

$$
Q=A / B \oplus C^{T} / A^{T}=\left[\begin{array}{ccc}
\frac{2}{3} & \frac{1}{9} & \frac{1}{3} \\
3 & 1 & \frac{4}{3} \\
1 & \frac{1}{2} & 0
\end{array}\right]
$$

and

$$
\Delta(Q)=\left[\begin{array}{ccc}
1 & \frac{1}{6} & \frac{1}{3} \\
3 & 1 & \frac{4}{3} \\
\frac{3}{2} & \frac{1}{2} & 1
\end{array}\right]
$$

Since $\operatorname{diag}(\Delta(Q))=I$, it follows that the diagonal matrix corresponding to any one of the three columns of $\Delta(Q)$ solves Problem 3.3 and the solution set consists of the non-zero max combination of these columns. For example if $u=\left[\begin{array}{lll}1 & 0 & \frac{9}{4}\end{array}\right]^{T}$ we obtain $x:=\Delta(Q) \otimes u=\left[\begin{array}{lll}1 & 3 & \frac{9}{4}\end{array}\right]^{T}$ and for $X=\operatorname{diag}(x)$ we have

$$
X^{-1} A X=\left[\begin{array}{ccc}
2 & 0 & 0 \\
3 & 1 & \frac{3}{2} \\
\frac{4}{3} & \frac{2}{3} & 0
\end{array}\right]
$$

Evidently $C \leq X^{-1} A X \leq B$. 
4. Full term rank scaling. The main aim of this section is to introduce and solve a new type of a scaling problem, formulated as Problem 4.6 below. For simplicity we first deal with an auxiliary problem (Problem 4.2) which, however, may be interesting on its own. Having dealt with a theoretical solution of Problem 4.6, we then describe an algorithm which finds it.

Given $A=\left(a_{i j}\right) \in \mathbb{R}_{+}^{n \times n}$ we put

$$
w(A, \pi)=a_{1, \pi(1)} a_{2, \pi(2)} \ldots a_{n, \pi(n)}
$$

for every permutation $\pi$ in the set $P_{n}$ of all permutations of $1, \ldots, n$. We will abbreviate $w(A, \pi)$ by $w(\pi)$ when it is clear to which matrix it refers. We will also use the following notations:

$$
\begin{aligned}
w^{*} & =w^{*}(A)=\max _{\pi \in P_{n}} w(A, \pi), \\
\operatorname{ap}(A) & =\left\{\pi \in P_{n} ; w^{*}=w(\pi)\right\} .
\end{aligned}
$$

We will be concerned with the permutations in $\operatorname{ap}(A)$, also called maximal permutations. We begin by stating some evident properties of the set $\operatorname{ap}(A)$, which are found in a slightly different terminology in [5].

Proposition 4.1. If $A, B \in \mathbb{R}_{+}^{n \times n}$ and $B=X A Y$ for some diagonal matrices $X, Y$ with positive diagonal, then

$$
w(B, \pi)=w(A, \pi) \operatorname{det} X \operatorname{det} Y
$$

for every $\pi \in P_{n}$ and hence

$$
\operatorname{ap}(B)=\operatorname{ap}(A)
$$

In particular,

$$
\operatorname{ap}\left(X^{-1} A X\right)=\operatorname{ap}(A)
$$

We now introduce our first problem in this section. In what follows the symbol $i d$ stands for the identity permutation.

Problem 4.2. Given $A=\left(a_{i j}\right) \in \mathbb{R}_{+}^{n \times n}$ with $a_{i i}>0, i=1, \ldots, n$, find a diagonal matrix $X$ with positive diagonal elements such that the scaling $X^{-1} A X=B=\left(b_{i j}\right)$ satisfies

$$
b_{i i}=\max _{j} b_{i j}=\max _{j} b_{j i} \text { for all } i=1, \ldots, n \text {. }
$$

Note that $b_{i i}=a_{i i}, i=1, \ldots, n$, if such a scaling exists.

Theorem 4.3. Let $A=\left(a_{i j}\right) \in \mathbb{R}_{+}^{n \times n}$ with $a_{i i}>0, i=1, \ldots, n$. Let $L=$ $\operatorname{diag}\left(a_{11}, \ldots, a_{n n}\right)$ and let

$$
Q=L^{-1} \otimes A \oplus A \otimes L^{-1}
$$


Then the following hold:

(a) $A$ diagonal similarity scaling $B=X^{-1} A X$ of $A$ that satisfies (4.2) exists if and only if the matrix $Q$ in (4.3) satisfies $\lambda(Q) \leq 1$ (or, equivalently, $\lambda(Q)=1$ ).

(b) If $\lambda(Q) \leq 1$, then $B=X^{-1} A X$ satisfies (4.2) if and only if

$$
X=\operatorname{diag}(x) \quad \text { for some } \quad x \in \operatorname{Comb}^{+}(\Delta(Q)) .
$$

Proof. Let $B \in \mathbb{R}_{+}^{n \times n}$ be defined by

$$
b_{i j}=\min \left\{a_{i i}, a_{j j}\right\}, \quad i, j=1, \ldots, n .
$$

Since $b_{i i}=a_{i i}, i=1, \ldots, n$, we require a scaling $X^{-1} A X$ such that $X^{-1} A X \leq B$. But by Corollary 3.6 and Lemma 2.2, Part 3, such a scaling exists if and only if $\lambda(A / B) \leq 1$. But it is easily proved that $A / B=Q$ where $Q$ is given by (4.3). Further, since $\operatorname{diag}(Q)=I$, it follows that $\lambda(Q) \leq 1$ is equivalent to $\lambda(Q)=1$. This proves (a).

Part (b) follows immediately by Corollary 3.6. $\square$

Remark 4.4. Since in Theorem $4.3 \operatorname{diag}(Q)=I$, we have $\Gamma(Q)=\Delta(Q)$. If $\lambda(Q)=1$ then by Condition (b) of Theorem 4.3 we may replace $x \in \operatorname{Comb}^{+}(\Delta(Q))$ either by $Q \otimes x \leq x, x>0$ or by $Q \otimes x=x, x>0$, see Corollary 2.9 and Proposition 2.5. Also note that if a solution to Problem 4.2 exists then $i d \in \operatorname{ap}(A)$. The converse is false as can be seen by the following example.

EXAMPLE 4.5. Let

$$
A=\left[\begin{array}{ll}
2 & 1 \\
2 & 1
\end{array}\right]
$$

Then

$$
Q=\left[\begin{array}{ll}
1 & 1 \\
2 & 1
\end{array}\right]
$$

Thus $\lambda(Q)>1$ and hence Problem 4.2 has no solution for this matrix.

Problem 4.6. Given $A=\left(a_{i j}\right) \in \mathbb{R}_{+}^{n \times n}, \alpha_{1}, \ldots, \alpha_{n}, \beta_{1}, \ldots, \beta_{n}>0$, find a matrix $B=\left(b_{i j}\right) \in \mathbb{R}_{+}^{n \times n}$ such that for some permutation $\pi \in P_{n}$

(a) $B=X A Y$ for some diagonal matrices $X, Y$ with positive diagonal,

(b) $B$ has row maxima $\alpha_{1}, \ldots, \alpha_{n}$, column maxima $\beta_{1}, \ldots, \beta_{n}$ and

(c) the entry $b_{i, \pi(i)}$ is both a column and row maximum for every $i \in N$.

We say that $B$ is a full term rank scaling of $A$ if $B$ satisfies all requirements of Problem 4.6.

Let $A \in \mathbb{R}_{+}^{n \times n}$ and suppose that $B$ is a full term rank scaling of $A$. Let $\pi$ be a permutation satisfying the Requirement (c) of Problem 4.6. It is easily seen then that $w^{*}(A)=w(\pi, A)>0, \pi \in \operatorname{ap}(B)=\operatorname{ap}(A)$ and that

$$
\alpha_{i}=\beta_{\pi(i)} \quad \text { for all } i=1, \ldots, n \text {. }
$$


We also have $\alpha_{i}=b_{i, \pi(i)}, i=1, \ldots, n$. At the same time, if $\sigma \in \operatorname{ap}(B)=\operatorname{ap}(A)$ then $b_{i, \pi(i)} \geq b_{i, \sigma(i)}$ for all $i=1, \ldots, n$ and

$$
\prod_{i=1}^{n} b_{i, \pi(i)}=\prod_{i=1}^{n} b_{i, \sigma(i)}
$$

and thus $b_{i, \pi(i)}=b_{i, \sigma(i)}$ for all $i=1, \ldots, n$, yielding that $b_{i, \sigma(i)}$ are row maxima for all $i=1, \ldots, n$. It is easily proved in a similar way that $b_{i, \sigma(i)}$ are column maxima for all $i=1, \ldots, n$. Therefore we have

TheOREM 4.7. Let $A \in \mathbb{R}_{+}^{n \times n}$ have a full term rank scaling $B$. Then the set of permutations $\pi$ satisfying $(4.4)$ is $\operatorname{ap}(A)$.

Now assume that $A \in \mathbb{R}_{+}^{n \times n}, w^{*}(A)>0$ and that the permutation $\pi \in \operatorname{ap}(A)$ satisfies (4.4). By Theorem 4.7, in order to determine the existence of a full term rank scaling of $A$ it is enough to determine whether (a) and (b) of Problem 4.6 can be satisfied for this particular permutation. Let $P$ be the permutation matrix corresponding to $\pi$ (viz. $p_{i j}=1$ if $j=\pi(i)$ and $p_{i j}=0$ otherwise) and let $Z=$ $\operatorname{diag}\left(\alpha_{1} / a_{1, \pi(1)}, \ldots, \alpha_{n} / a_{n, \pi(n)}\right)$. Then the matrix $C:=A P^{-1} Z$ has $c_{i i}=\alpha_{i}, i=$ $1, \ldots, n$. It follows that there exists a full term rank scaling of $A$ if and only if there exists a similarity scaling $B=X^{-1} C X$ of $C$ satisfying condition (4.2) in Problem 4.2. In this case, a full term rank scaling of $A$ is given by $X^{-1} A P^{-1} Z X P$.

This leads to the following algorithm. We note that Step 1 is essentially solving the assignment problem for which solution methods may be found in many standard texts, e.g. [21, Chapter 5], [17] and [22, pp. 248-255].

\section{ALGORITHM FULL TERM RANK SCALING}

Input: $A=\left(a_{i j}\right) \in \mathbb{R}_{+}^{n \times n}, \alpha_{1}, \ldots, \alpha_{n}, \beta_{1}, \ldots, \beta_{n}>0$.

Output: $B=\left(b_{i j}\right) \in \mathbb{R}_{+}^{n \times n}$ such that $B=X A Y$ for some diagonal matrices $X$ and $Y$ with positive diagonal, $B$ having row maxima $\alpha_{1}, \ldots, \alpha_{n}$, column maxima $\beta_{1}, \ldots, \beta_{n}$ and for some permutation $\pi \in P_{n}$ the entry $b_{i, \pi(i)}$ is both a column and row maximum (for every $i \in N$ ); or an indication that no such matrix exists.

[1] Find $\pi \in \operatorname{ap}(A)$. If $w(\pi)=0$ then $\operatorname{STOP}\left(w^{*}(A)=0, B\right.$ does not exist $)$.

[2] Check that $\alpha_{i}=\beta_{\pi(i)}, i=1, \ldots, n$. Else $\operatorname{STOP}$ ( $B$ does not exist).

[3] Permute columns (or rows) of $A$ so that $\pi=i d$. Denote the new matrix by $A$.

[4] Scale the columns (or rows) of $A$ so that $a_{i i}=\alpha_{i}(i=1, \ldots, n)$. Denote the new matrix by $C$.

[5] Set $L=\operatorname{diag}\left(\alpha_{1}, \ldots, \alpha_{n}\right)$ and $Q=L^{-1} \otimes C \oplus C \otimes L^{-1}$.

[6] If $\lambda(Q)>1$ then $\operatorname{STOP}(B$ does not exist).

[7] Compute $x:=\Delta(Q) \otimes u$ where $u \geq 0, u \in \mathbb{R}_{+}^{n}$ and $x>0$. (Note that it suffices to take any $u>0$ or, if $\Delta(Q)>0$, we may take $u \geq 0, u \neq 0$.) Let $X=\operatorname{diag}(x)$.

[8] Set $D=X^{-1} C X$ and apply to $D$ the column (or row) permutation inverse to the one used in Step 3. The resulting matrix is $B$. 
EXAMPLE 4.8. Let

$$
A=\left[\begin{array}{ccc}
2 & 1 & 6 \\
12 & 6 & 36 \\
3 & 3 & 9
\end{array}\right]
$$

and suppose that $\left(\alpha_{1}, \alpha_{2}, \alpha_{3}\right)=(2,2,1)$ and $\left(\beta_{1}, \beta_{2}, \beta_{3}\right)=(2,1,2)$. Then $w^{*}(A)=$ 216 and $\pi$ given by $\pi(1)=3, \pi(2)=1, \pi(3)=2$ is one of two permutations in $\operatorname{ap}(A)$. Thus Steps 1 and 2 allow us to proceed. Using this permutation we obtain at Step 3

$$
\left[\begin{array}{ccc}
6 & 2 & 1 \\
36 & 12 & 6 \\
9 & 3 & 3
\end{array}\right]
$$

at Step 4

$$
C=\left[\begin{array}{ccc}
2 & \frac{1}{3} & \frac{1}{3} \\
12 & 2 & 2 \\
3 & \frac{1}{2} & 1
\end{array}\right]
$$

and at Step 5

$$
Q=\left[\begin{array}{lll}
1 & \frac{1}{6} & \frac{1}{3} \\
6 & 1 & 2 \\
3 & \frac{1}{2} & 1
\end{array}\right]
$$

Clearly $\lambda(Q)=1$, so computing $\Delta(Q) \otimes u$ with $u=[1,0,0]^{T}$ we obtain in Step 7

$$
X=\operatorname{diag}(1, \quad 6, \quad 3)
$$

and finally in Step 8 we obtain the full term rank scaling

$$
B=\left[\begin{array}{lll}
2 & 1 & 2 \\
2 & 1 & 2 \\
1 & 1 & 1
\end{array}\right]
$$

EXAMPLE 4.9. Let

$$
A=\left[\begin{array}{ll}
1 & 1 \\
1 & 1
\end{array}\right]
$$

and suppose that $\left(\alpha_{1}, \alpha_{2}\right)=\left(\beta_{1}, \beta_{2}\right)=(1,2)$. Let $\pi=i d$. Then the requirements of Steps 1 and 2 are satisfied, but at Step 5 we obtain

$$
Q=\left[\begin{array}{ll}
1 & 2 \\
1 & 2
\end{array}\right]
$$

Since $\lambda(Q)>1$, no full term rank scaling of $A$ exists. Note that the permutation (2 1 ) does not satisfy the requirement of Step 2. This example also shows that the hypothesis of Theorem 4.7 cannot be omitted. 


\section{REFERENCES}

[1] F. L. Baccelli, G. Cohen, G.-J. Olsder and J.-P. Quadrat. Synchronization and Linearity. Chichester, New York, J.Wiley and Sons, 1992.

[2] R. B. Bapat. A max version of the Perron-Frobenius Theorem. Linear Algebra and its Applications, 275/276:3-18, 1998.

[3] R. B. Bapat and T. E. S. Raghavan. Nonnegative Matrices and Applications. Cambridge University Press, 1997.

[4] R. B. Bapat, D. P. Stanford, and P. van den Driessche. Pattern properties and spectral inequalities in max algebra. SIAM Journal on Matrix Analysis and Applications, 16:964-976, 1995.

[5] R. E. Burkard and E. Çela. Linear Assignment Problems and Extensions. Handbook of Combinatorial Optimization (P.M.Pardalos and D.-Z.Du, Eds.), Supplement Volume A, Kluwer Academic Publishers, 75-149, 1999.

[6] P. Butkovič. Max-algebra: the linear algebra of combinatorics? Linear Algebra and its Applications, 367:313-335, 2003.

[7] R. A. Cuninghame-Green. Minimax Algebra. Lecture Notes in Economics and Mathematical Systems 166, Berlin, Springer, 1979.

[8] R. A. Cuninghame-Green. Minimax algebra and applications. Advances in Imaging and Electron Physics, Vol. 90, pp. 1-121, Academic Press, New York, 1995.

[9] L. Elsner and P. van den Driessche. On the power method in max algebra. Linear Algebra and its Applications, 302/303:17-32, 1999.

[10] L. Elsner and P. van den Driessche. Modifying the power method in max algebra. Linear Algebra and its Applications, 332/334:3-13, 2001.

[11] G. M. Engel and H. Schneider. Cyclic and Diagonal Products on a Matrix. Linear Algebra and its Applications, 7:301-335, 1973.

[12] G. M. Engel and H. Schneider. Diagonal similarity and equivalence for matrices over groups with 0. Czechoslovak Mathematical Journal, 25:389-403, 1975.

[13] M. Fiedler and V. Pták. Diagonally dominant matrices. Czechoslovak Mathematical Journal, 92:420-433, 1967.

[14] M. Fiedler and V. Pták. Cyclic products and an inequality for determinants. Czechoslovak Mathematical Journal, 19:428-450, 1969.

[15] S. Gaubert. Théorie des systèmes linéaires dans les diö̈des. Thèse. Ecole des Mines de Paris, 1992.

[16] S. Gaubert et al. Algèbres Max-Plus et applications en informatique et automatique. 26ème école de printemps d'informatique théorique, Noirmoutier, 1998.

[17] M. Gondran. Path algebra and algorithms. Combinatorial programming: methods and applications. Proc. NATO Advanced Study Inst., Versailles, 1974), (B. Roy, Ed.), NATO Advanced Study Inst. Ser., Ser. C: Math. and Phys. Sci. 19, Dordrecht, Reidel, pp. 137-148, 1975.

[18] M. Gondran and M. Minoux. Linear algebra of diö̈ds: a survey of recent results. Annals of Discrete Mathematics, 19:147-164, 1984.

[19] D. Hershkowitz and H. Schneider. One-sided simultaneous inequalities and sandwich theorems for diagonal similarity and diagonal equivalence of nonnegative matrices. Electronic Journal of Linear Algebra, 10:81- 101, 2003.

[20] R. M. Karp. A characterization of the minimum cycle mean in a digraph. Discrete Mathematics, $23: 309-311,1978$

[21] E. L. Lawler. Combinatorial optimization, Networks and Matroids. Holt, Rinehart, Winston, 1976.

[22] C. H. Papadimitriou and K. Steiglitz. Combinatorial Optimization-Algorithms and Complexity. Dover, 1998.

23] B.D. Saunders and H. Schneider. Flows of graphs applied to diagonal similarity and equivalence of matrices. Discrete Mathematics, 24:139-162, 1978.

[24] U. Zimmermann. Linear and Combinatorial Optimization in Ordered Algebraic Structures. Annals of Discrete Mathematics, 10, Amsterdam, North Holland, 1981. 